\title{
Florística do estrato herbáceo de um remanescente de cerradão em Campo Grande, Mato Grosso do Sul, Brasil ${ }^{1}$
}

\author{
Vivian Almeida Assunção ${ }^{2,3}$, Adriana Guglieri-Caporal ${ }^{2}$ e Ângela Lúcia Bagnatori Sartori ${ }^{2}$
}

Recebido: 20.08.2009; aceito: 22.06.2011

\begin{abstract}
Floristic survey of the herbaceous layer of a cerrado woodland remnant in Campo Grande, Mato Grosso do Sul, Brazil). The Cerrado is the second largest Brazilian morphoclimatic domain. It very rich in species, many endemic but it is also influenced by surrounding phytogeographic domains. The Cerrado, particularly cerrado woodland, is vulnerable to changes in climate and soil and to wildfires. Based on the avaliation of the richness of the herbaceous of the cerrado woodland of an urban RPPN want to answer the following questions: What is the growth habit, biological form and dispersal syndrome predominant? These species are exclusive of Cerrado phytogeographical domain? Samples were collected from May 2007 to June 2008 in a remnant of cerrado woodland on the RPPN/UFMS, Campo Grande, MS. We found 59 species, 49 genera and 17 families. The families most representative were Fabaceae (15 species), Poaceae (12) and Asteraceae (seven). Predominated the erect herbs $(83 \%)$, hemicryptophyte $(59 \%)$ and autochorich $(61 \%)$. About distribution of species in Brazilian phytogeographic domains, $12 \%$ are exclusive to Cerrado and $16 \%$ are in Cerrado, Amazônia, Caatinga, Mata Atlântica and Pampa.
\end{abstract}

Key words: flora, floristic survey, herbs, savannah

RESUMO - (Florística do estrato herbáceo de um remanescente de cerradão em Campo Grande, Mato Grosso do Sul, Brasil). O Cerrado é o segundo maior domínio morfoclimático brasileiro. O Cerrado é o segundo maior domínio morfoclimático brasileiro. Esta formação é rica em espécies, recebe influência dos domínios fitogeográficos circunvizinhos. O Cerrado, particularmente o cerradão, é vulnerável às mudanças de clima, solo e queimadas. Com base na avaliação da riqueza do estrato herbáceo do cerradão de uma RPPN urbana pretende-se responder as seguintes questões: Qual o hábito de crescimento, forma biológica e síndrome de dispersão predominantes? As espécies avaliadas são exclusivas do domínio fitogeográfico do Cerrado? As coletas foram realizadas de maio de 2007 a junho de $2008 \mathrm{em}$ um remanescente de cerradão da RPPN/UFMS, Campo Grande, MS. Encontramos 59 espécies, 49 gêneros e 17 famílias. As famílias mais representativas foram Fabaceae (15 espécies), Poaceae (12) e Asteraceae (sete). Predominaram as herbáceas eretas (83\%), hemicriptófitas (59\%) e autocóricas (61\%). Em relação à distribuição nos domínios fitogeográficos brasileiros observamos que $12 \%$ das espécies são exclusivas de Cerrado e 16\% ocorrem no Cerrado, Amazônia, Caatinga, Mata Atlântica e Pampa.

Palavras-chave: Cerrado, ervas, flora, levantamento florístico

\section{Introdução}

O Cerrado é o segundo maior domínio morfoclimático brasileiro (Rodrigues 2005) e sua flora estimada em cerca de 12.000 espécies é formada predominantemente pelo estrato herbáceo (Mendonça et al. 2008). Sua composição florística varia entre as fitofisionomias em função do gradiente de luz e tolerância ao sombreamento (Coutinho 1978, Mantovani \& Martins 1993) e é vulnerável às mudanças de clima, tipos de solo e ocorrência de queimadas (Batalha \& Martins 2002).

Estudos florísticos realizados no Cerrado estão restritos aos estratos arbóreo e arbustivo, sendo o herbáceo e o subarbustivo comumente negligenciados. Dentre os estudos florísticos envolvendo o estrato herbáceo no Cerrado destacam-se os de: Tannus \& Assis (2004) em campo úmido, Munhoz \& Felfili (2005, 2006) em campo sujo, Carvalho (1993), Mantovani \& Martins (1993), Batalha et al. (2001),

1. Parte da Monografia de Conclusão de Curso de Ciências Biológicas da primeira autora na Universidade Federal de Mato Grosso do Sul

2. Universidade Federal de Mato Grosso do Sul, CCBS/DBI, Laboratório de Botânica, Cidade Universitária, Caixa Postal 549, 79070-900 Campo Grande, MS, Brasil

3. Autor para correspondência: vivian.bios@gmail.com 
Batalha \& Mantovani (2001), Batalha \& Martins (2002), Meira Neto et al. (2007) e Mendonça et al. (2007) em diferentes formações vegetacionais.

A avaliação das formas biológicas e do hábito de crescimento tem sido utilizada para descrever qualitativamente a fisionomia da vegetação, a partir da quantificação de suas categorias (Mueller-Dombois \& Ellenberg 1974).

Os mecanismos de dispersão, essenciais na distribuição natural das espécies, requer interações entre as plantas e seus dispersores e podem variar conforme os hábitos de crescimento. A dispersão de espécies no Cerrado pode ser efetuada por animais em espécies arbóreas ou por agentes abióticos em espécies herbáceas e subarbustivas (Batalha \& Martins 2004).

Determinados estudos (Prado et al. 1992, Oliveira Filho \& Ratter 1995) tem indicado que o Cerrado influencia e recebe influência de distintos domínios fitogeográficos brasileiros compartilhando com esses alguns táxons. Entretanto, a distribuição geográfica dos táxons herbáceos ocorrentes no Cerrado e nos diferentes domínios brasileiros ainda requer análises.

O presente estudo com base na avaliação da riqueza do estrato herbáceo do cerradão de uma RPPN urbana pretende responder as seguintes questões: Qual o hábito de crescimento, a forma biológica e a síndrome de dispersão predominantes no cerradão? As espécies avaliadas são exclusivas do domínio fitogeográfico do Cerrado?

\section{Material e métodos}

O estudo foi realizado na Reserva Particular do Patrimônio Natural da Universidade Federal de Mato Grosso do Sul (RPPN/UFMS), cadastrada desde fevereiro de 2003 no Sistema Nacional de Unidades de Conservação. A RPPN com 50 ha, se localiza em Campo Grande, Mato Grosso do Sul (figura 1a), nas coordenadas geográficas $20^{\circ} 30^{\prime} \mathrm{S}$ e $54^{\circ} 36^{\prime} \mathrm{W}$, a $600 \mathrm{~m}$ de altitude. O clima da região é o Cwa conforme classificação de Köppen (1931), com temperatura média entre 20 e $22{ }^{\circ} \mathrm{C}$, com alternância de duas estações, chuvosa (verão) e seca (inverno), a precipitação média anual é de $1.532 \mathrm{~mm}$ e a umidade relativa raramente atinge $80 \%$ (Embrapa-CNPGC 1985). O solo é caracterizado como Latossolo Vermelho Escuro, Latossolo Roxo, Areia Quartzosas e solos litólicos (REPAMS 2008).
A formação fitofisionômica da RPPN/UFMS foi classificada como cerradão de acordo com Furley \& Ratter (1988), constituído por três estratos: o superior, com árvores de 10-12 m de altura e algumas emergentes de 15-18 m; o mediano, mais ou menos denso, formado por arbustos ou arvoretas que atingem até $3 \mathrm{~m}$ e o estrato herbáceo. É observada a ocorrência de clareiras em trechos da área de estudo, localizadas no interior das parcelas. Atualmente encontra-se em regeneração, tendo sido por anos sujeita a pastejo de gado bovino.

A área selecionada para estudo com cerca de 3 ha corresponde a uma parte da Reserva (figura 1b), onde foram estabelecidas 13 parcelas, plotadas no sentido leste-oeste, de $2.400 \mathrm{~m}^{2}(400 \times 6 \mathrm{~m}$ cada $)$, distantes a $29 \mathrm{~m}$ uma da outra.

As coletas mensais foram realizadas no período de maio de 2007 a junho de 2008 por meio de caminhadas nas parcelas. Durante as coletas, foram obtidos indivíduos preferencialmente férteis. A definição do hábito herbáceo seguiu Guedes-Bruni et al. (2002), onde foram consideradas ervas as plantas não lenhosas.

Para identificação do material coletado foram utilizadas bibliografias especializadas, comparação com material de herbário e quando necessário consulta a especialistas. A nomenclatura para família seguiu APG III (2009). O material fértil coletado e devidamente identificado foi herborizado de acordo com as técnicas usuais e incorporado ao Herbário CGMS da Universidade Federal de Mato Grosso do Sul campus Campo Grande.

O hábito de crescimento dos indivíduos amostrados foi classificado em ereto, subereto, decumbente, prostrado, ereto e decumbente, e ereto a subereto, a partir de observações em campo. Cada espécie foi classificada quanto à forma biológica (caméfita, geófita, hemicriptófita, terófita, terófita a hemicriptófita), de acordo com o sistema de Raunkier adaptado por Müller-Dombois \& Ellenberg (1974).

A caracterização das síndromes de dispersão foi baseada no tamanho, cor e morfologia dos diásporos, incluindo a ornamentação e estruturas acessórias. Para as síndromes foi adotada a nomenclatura de van der Pijl (1982), sendo consideradas as categorias autocórica, anemocórica e zoocórica.

Informações referentes à distribuição das espécies quanto aos domínios fitogeográficos brasileiros foram compilados de literatura disponível e da Lista de Espécies da Flora do Brasil (http://floradobrasil.jbrj.gov.br/2010/); não foi 


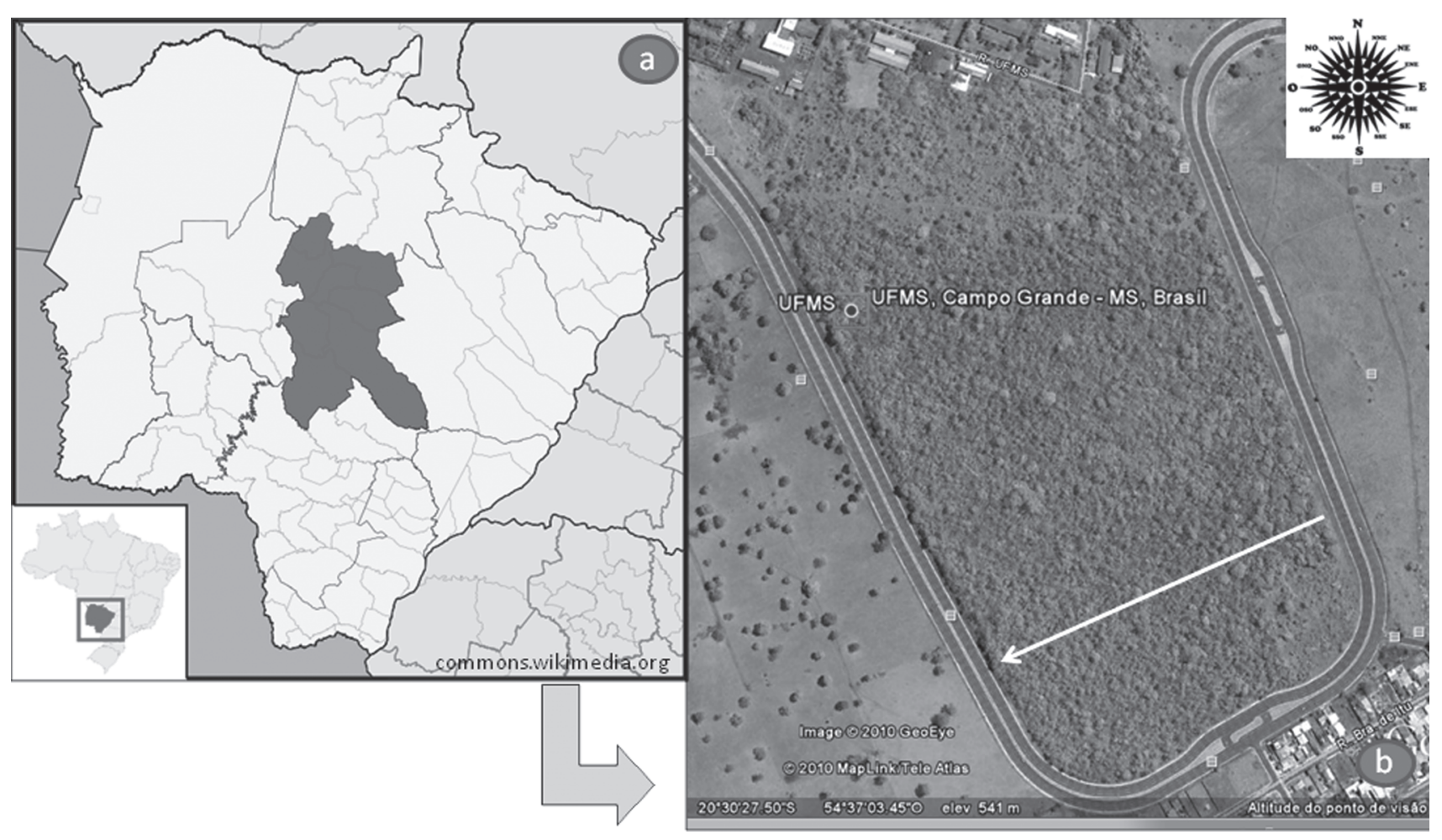

Figura 1. a. Mapa de Mato Grosso do Sul com destaque para o município de Campo Grande. b. Localização e vista aérea da Reserva Particular do Patrimônio Natural da Universidade Federal de Mato Grosso do Sul (RPPN/UFMS) campus Campo Grande, Mato Grosso do Sul. A seta mostra o sentido no qual as parcelas foram alocadas na área.

Figure 1. a. Map of Mato Grosso do Sul, especially the city of Campo Grande. b. Location and aerial view of Reserva Particular do Patrimônio Natural da Universidade Federal de Mato Grosso do Sul (RPPN/UFMS) campus Campo Grande, Mato Grosso do Sul. The arrow shows the direction in which the plots were allocated in the area

considerado o domínio fitogeográfico do Pantanal como unidade diferenciada conforme proposto por Joly et al. (1999). Indivíduos identificados ao nível de gênero não foram considerados nesta análise.

\section{Resultados}

Foram encontradas 59 espécies, distribuídas em 49 gêneros e 17 famílias (tabela 1); Fabaceae foi a mais rica em espécies (15 espécies), seguida por Poaceae (12) e Asteraceae (sete) que representam juntas cerca de $58 \%$ do total amostrado.

O gênero com maior riqueza foi Mimosa com três espécies, seguido de Arachis, Aristida, Desmodium, Eriosema, Gomphrena, Hybanthus, Pavonia e Sida, com duas espécies cada. Dentre as espécies constatadas, $83 \%$ foram classificadas como eretas e as demais categorias variaram pouco entre si; hemicriptófita foi a forma de vida predominante (ca. $59 \%$ ) e geófita a mais rara (ca. 8\%), como mostra a tabela 1. Observamos a ocorrência de um grande número de heliófilas em clareiras e na borda do cerradão.
As síndromes de dispersão mais comuns entre as espécies avaliadas foi a autocórica (ca. 61\%) e a anemocórica (ca. 24\%). O predomínio de espécies autocóricas deve-se principalmente aos representantes de Fabaceae e Poaceae, que juntos somaram aproximadamente 56\% desta categoria. Asteraceae contribuiu consideravelmente com o predomínio de espécies anemocóricas (ca. 43\%). As espécies destas famílias foram mais comuns na borda e em clareiras dentro da área de estudo. Mimosa maracayuensis e Hybanthus spp. não foram classificados quanto à síndrome de dispersão devido à ausência de frutos.

Das espécies avaliadas quanto à distribuição nos domínios fitogeográficos brasileiros, $12 \%$ são exclusivas do Cerrado e $16 \%$ ocorrem nos cinco domínios considerados. Das 49 espécies avaliadas, constatamos maior número de espécies em comum com a Mata Atlântica (37 espécies) e menor número com o Pampa (12) como mostra a tabela 2.

Alysicarpus vaginalis, Oeceoclades maculata, Digitaria insularis, Hyparrhenia rufa, Melinis minutiflora e Urochloa brizantha são consideradas 
Tabela 1. Espécies herbáceas encontradas na RPPN/UFMS, Campo Grande, Mato Grosso do Sul. Informações sobre hábito de crescimento dos indivíduos, forma de vida, síndromes de dispersão e material testemunho (Herbário CGMS da Universidade Federal de Mato Grosso do Sul).

Table 1. Herbaceous species found in the RPPN/UFMS, Campo Grande, Mato Grosso do Sul information about growth habit, life form, dispersal syndromes and voucher (CGMS Herbarium da Universidade Federal de Mato Grosso do Sul).

\begin{tabular}{|c|c|c|c|c|}
\hline Famíla/Subfamília/Espécie & $\begin{array}{c}\text { Hábito de } \\
\text { crescimento }\end{array}$ & $\begin{array}{l}\text { Forma de } \\
\text { vida }\end{array}$ & $\begin{array}{l}\text { Síndrome de } \\
\text { dispersão }\end{array}$ & $\begin{array}{c}\text { Material } \\
\text { testemunho } \\
\text { (CGMS) }\end{array}$ \\
\hline
\end{tabular}

\section{ACANTHACEAE}

Justicia sp.

Ruellia cf. geminiflora Kunth

Stenandrium pohli Nees

AMARANTHACEAE

Gomphrena macrocephala A. St.-Hil.

Gomphrena sp.

ASTERACEAE

Acanthospermum australe (Loefl.) Kuntze

Elephantopus angustifolius Sw.

Eupatorium sp.

Porophyllum ruderale (Jacq.) Cass.

Pterocaulon lanatum Kuntze

Tridax procumbens L.

Vernonia remotiflora Rich.

BROMELIACEAE

Ananas ananassoides (Baker) L.B. Sm.

Dyckia sp.

COMMELINACEAE

Commelina erecta L.

CYPERACEAE

Cyperus imbricatus Retz.

Rhynchospora nervosa (Vahl) Boeckeler

EUPHORBIACEAE

Cnidoscolus sp.

Euphorbia hyssopifolia L.

IRIDACEAE

Cipura paludosa Aubl.

FABACEAE

Caesalpinoideae

Chamaecrista nictitans (L.) Moench

Mimosoideae

Mimosa diplotricha C. Wright ex Sauvalle

Mimosa gracilis Benth.

Mimosa maracayuesis Chodat \& Hassl.

Papilionoideae

Alysicarpus vaginalis (L.) DC.

Arachis archeri Krapov. \& W.C. Gregory

Arachis glabrata Benth.

Clitoria falcata Lam.

Crotalaria stipularia Desv.

Desmodium barbatum (L.) Benth.

Desmodium incanum (Sw.) DC.

Eriosema campestre Benth.

Eriosema crinitum (Kunth) G. Don

Periandra heterophylla Benth.

Zornia latifolia Sm.

LYTHRACEAE

Cuphea carthagenensis (Jacq.) J.F. Macbr.

Ereta
Ereta
Ereta
Ereta
Ereta
Prostrada
Ereta
Ereta
Ereta
Ereta
Prostrada
Ereta
Ereta
Ereta

Hemicriptófita
Hemicriptófita
Hemicriptófita
Hemicriptófita
Geófita
Terófita
Hemicriptófita
Hemicriptófita
Terófita
Terófita/Hemicriptófita
Terófita
Indeterminada
Hemicriptófita

Ereta

Ereta

Ereta

Ereta

Ereta

Ereta

Ereta

Ereta

Ereta

Ereta

Ereta

Decumbente

Prostrada

Ereta

Ereta

Ereta e decumbente

Ereta e decumbente

Ereta

Ereta

Ereta

Decumbente

Ereta

Hemicriptófita

Terófita

Hemicriptófita

Hemicriptófita

Terófita

Geófita

Caméfita

Hemicriptófita

Hemicriptófita

Caméfita

Geófita

Geófita

Caméfita

Hemicriptófita

Hemicriptófita

Hemicriptófita

Hemicriptófita

Hemicriptófita

Caméfita

Hemicriptófita

Terófita
Autocórica

23987

Autocórica $\quad 23988$

Autocórica 23989

Anemocórica $\quad 23990$

Anemocórica 23991

Zoocórica 23992

Anemocórica 23993

Anemocórica 23994

Anemocórica 23995

Anemocórica 23996

Anemocórica 23997

Anemocórica 23998

Zoocórica 23999

Anemocórica

24000

Autocórica

24001

Autocórica $\quad 24002$

Autocórica 24003

Autocórica

24005

Autocórica

24004

Autocórica

24006

Autocórica

24007

Autocórica

24008

Autocórica

29511

24009

Autocórica

24010
Autocórica

Autocórica

Autocórica

24012

24011

24013

24014

24015

24016

24017

24018

24019

24020

Autocórica

Zoocória

24021

Autocórica 


\begin{tabular}{|c|c|c|c|c|}
\hline Famíla/Subfamília/Espécie & $\begin{array}{l}\text { Hábito de } \\
\text { crescimento }\end{array}$ & $\begin{array}{l}\text { Forma de } \\
\text { vida }\end{array}$ & $\begin{array}{l}\text { Síndrome de } \\
\text { dispersão }\end{array}$ & $\begin{array}{c}\text { Material } \\
\text { testemunho } \\
\text { (CGMS) }\end{array}$ \\
\hline \multicolumn{5}{|l|}{ MALVACEAE } \\
\hline Pavonia communis A. St.-Hil. & Ereta & Caméfita & Zoocórica & 24022 \\
\hline Pavonia sp. & Ereta & Hemicriptófita & Autocórica & 24023 \\
\hline Sida cordifolia $\mathrm{L}$. & Ereta & Hemicriptófita & Autocórica & 24024 \\
\hline Sida rhombifolia $\mathrm{L}$. & Ereta & Terófita/Hemicriptófita & Autocórica & 24025 \\
\hline Waltheria communis A. St.-Hil. & Ereta & Caméfita & Autocórica & 24026 \\
\hline \multicolumn{5}{|l|}{ ORCHIDACEAE } \\
\hline Oeceoclades maculata (Lindl.) Lindl. & Ereta & Caméfita & Anemocórica & 24027 \\
\hline \multicolumn{5}{|l|}{ POACEAE } \\
\hline Andropogon fastigiatus Sw. & Ereta & Terófita & Autocórica & 24028 \\
\hline Aristida riparia Trin. & Ereta & Hemicriptófita & Anemocórica & 24053 \\
\hline Aristida setifolia Kunth & Ereta & Hemicriptófita & Anemocórica & 24029 \\
\hline Axonopus pressus (Ness ex Steud.) Parodi & Ereta & Hemicriptófita & Autocórica & 24030 \\
\hline *Digitaria insularis (L.) Fedde & Ereta & Hemicriptófita & Autocórica & 24031 \\
\hline Eragrostis polytricha Ness & Ereta & Hemicriptófita & Autocórica & 24032 \\
\hline Hyparrhenia rufa (Ness) Stapf & Ereta & Hemicriptófita & Autocórica & 24034 \\
\hline Ichnanthus procurrens (Ness ex Trin.) Swallen & Ereta e decumbente & Hemicriptófita & Autocórica & 24035 \\
\hline Melinis minutiflora P. Beauv. & Ereta e decumbente & Hemicriptófita & Anemocórica & 24036 \\
\hline Paspalum glaucescens Hack. & Ereta & Hemicriptófita & Autocórica & 24037 \\
\hline Schizachyrium $\mathrm{sp}$. & Ereta & Hemicriptófita & Anemocórica & 24038 \\
\hline $\begin{array}{l}\text { Urochloa brizantha (Hochst. ex A. Rich.) R.D. } \\
\text { Webster }\end{array}$ & Ereta e subereta & Hemicriptófita & Autocórica & 24039 \\
\hline \multicolumn{5}{|l|}{ POLYGALACEAE } \\
\hline Polygalasp. & Ereta & Terófita & Autocórica & 24040 \\
\hline \multicolumn{5}{|l|}{ SOLANACEAE } \\
\hline Solanum sisymbrifolium Lam. & Ereta & Terófita & Zoocórica & 24041 \\
\hline \multicolumn{5}{|l|}{ TURNERACEAE } \\
\hline Piriqueta corumbensis Moura & Ereta & Geófita & Autocórica & 24042 \\
\hline \multicolumn{5}{|l|}{ VIOLACEAE } \\
\hline Hybanthus sp. 1 & Ereta & Hemicriptófita & Indeterminada & 24043 \\
\hline Hybanthus sp. 2 & Ereta & Hemicriptófita & Indeterminada & 24057 \\
\hline
\end{tabular}

como subespontâneas, o que corresponde a $12 \%$ das espécies avaliadas (tabela 2).

\section{Discussão}

Em uma área de cerradão em Santa Bárbara, SP, foram constatadas duas espécies herbáceas (Meira Neto et al. 2007) e em Assis, SP, 14 (Rossato et al. 2008), números nitidamente inferiores ao obtido pelo presente estudo. Possivelmente diferenças nos métodos de amostragem e esforço amostral tenham sido responsáveis por tais discrepâncias.

Fabaceae se destacou em riqueza no estrato herbáceo de cerradão em Campo Grande, MS (Cristaldo dados não publicados), assim como em cerrado denso em Moji Guaçu, SP (Mantovani \& Martins 1993). Semelhantes ao presente estudo, as áreas de cerrado avaliadas pelos referidos autores são atualmente protegidas e estão em regeneração, após terem sido, ao longo de anos, desmatadas e queimadas para implantação de pastagens.

Filgueiras (2002) observou que Fabaceae e Asteraceae estão entre as famílias mais representativas nas comunidades herbáceas de cerradão. Estudos anteriores destacaram como famílias de maior riqueza florística, Cyperaceae e Poaceae em campo úmido (Tannus \& Assis 2004), Poaceae e Asteraceae em vereda (Araújo et al. 2002), e Poaceae em campo sujo (Munhoz \& Felfili 2004, 2006). Já o elevado número de espécies de gramíneas no presente estudo se deve em parte à alteração da área, que propiciou o estabelecimento de espécies oportunistas como Andropogon fastigiatus, Digitaria insularis, Hyparrhenia rufa, Melinis minutiflora e Urochloa brizantha.

Eriosema e Mimosa se destacaram entre os gêneros mais representativos nos estudos de Carvalho 
Tabela 2. Distribuição das espécies herbáceas encontradas na RPPN/UFMS, Campo Grande, Mato Grosso do Sul, conforme os domínios fitogeográficos brasileiros. *espécies subespontâneas.

Table 2. Distribution of herbaceous species found in the RPPN/UFMS, Campo Grande, Mato Grosso do Sul, according to the Brazilian phytogeographic areas. *subspontaneous species.

\begin{tabular}{|c|c|c|c|c|c|}
\hline Família/Subfamília/Espécie & Amazônia & Caatinga & Cerrado & Mata Atlântica & Pampa \\
\hline \multicolumn{6}{|l|}{ ACANTHACEAE } \\
\hline Ruellia cf. gemminiflora & $X$ & $\mathrm{X}$ & $X$ & $\mathrm{X}$ & \\
\hline Stenandrium pohli & & & $\mathrm{X}$ & $\mathrm{X}$ & \\
\hline \multicolumn{6}{|l|}{ AMARANTHACEAE } \\
\hline Gomphrena macrocephala & & & $\mathrm{X}$ & & \\
\hline \multicolumn{6}{|l|}{ ASTERACEAE } \\
\hline Acanthospermum australe & $\mathrm{X}$ & & $X$ & $X$ & $\mathrm{X}$ \\
\hline Elephantopus angustifolius & $\mathrm{X}$ & $\mathrm{X}$ & $\mathrm{X}$ & $\mathrm{X}$ & $\mathrm{X}$ \\
\hline Porophyllum ruderale & $X$ & $X$ & $\mathrm{X}$ & & \\
\hline Pterocaulon lanatum & & & $\mathrm{X}$ & & \\
\hline Tridax procumbens & $X$ & $\mathrm{X}$ & $\mathrm{X}$ & $X$ & $\mathrm{X}$ \\
\hline Vernonia remotiflora & & & $\mathrm{X}$ & & \\
\hline \multicolumn{6}{|l|}{ BROMELIACEAE } \\
\hline Ananas ananassoides & $\mathrm{X}$ & $\mathrm{X}$ & $\mathrm{X}$ & $\mathrm{X}$ & \\
\hline \multicolumn{6}{|l|}{ COMMELINACEAE } \\
\hline Commelina erecta & $X$ & $X$ & $\mathrm{X}$ & $\mathrm{X}$ & \\
\hline \multicolumn{6}{|l|}{ CYPERACEAE } \\
\hline Cyperus imbricatus & $X$ & $X$ & $\mathrm{X}$ & $X$ & $\mathrm{X}$ \\
\hline Rhynchospora nervosa & $\mathrm{X}$ & $\mathrm{X}$ & $\mathrm{X}$ & $\mathrm{X}$ & \\
\hline \multicolumn{6}{|l|}{ EUPHORBIACEAE } \\
\hline Euphorbia hyssopifolia & $\mathrm{X}$ & $\mathrm{X}$ & $\mathrm{X}$ & $\mathrm{X}$ & $\mathrm{X}$ \\
\hline \multicolumn{6}{|l|}{ IRIDACEAE } \\
\hline Cipura paludosa & $\mathrm{X}$ & $\mathrm{X}$ & $\mathrm{X}$ & $X$ & \\
\hline \multicolumn{6}{|l|}{ FABACEAE } \\
\hline \multicolumn{6}{|l|}{ Caesalpinoideae } \\
\hline Chamaecrista nictitans & $\mathrm{X}$ & $X$ & $\mathrm{X}$ & $\mathrm{X}$ & \\
\hline \multicolumn{6}{|l|}{ Mimosoideae } \\
\hline Mimosa diplotricha & $\mathrm{X}$ & & $\mathrm{X}$ & $\mathrm{X}$ & \\
\hline Mimosa gracilis & & & $\mathrm{X}$ & $\mathrm{X}$ & \\
\hline Mimosa maracayüensis & & & $\mathrm{X}$ & $\mathrm{X}$ & \\
\hline \multicolumn{6}{|l|}{ Papilionoideae } \\
\hline * Alysicarpus vaginalis & & & $\mathrm{X}$ & & \\
\hline Arachis archeri & & & $\mathrm{X}$ & & \\
\hline Arachis glabrata & & & $\mathrm{X}$ & $\mathrm{X}$ & \\
\hline Clitoria falcata & $\mathrm{X}$ & & $\mathrm{X}$ & $\mathrm{X}$ & \\
\hline Crotalaria stipularia & $\mathrm{X}$ & $\mathrm{X}$ & $\mathrm{X}$ & $\mathrm{X}$ & \\
\hline Desmodium barbatum & $\mathrm{X}$ & $\mathrm{X}$ & $\mathrm{X}$ & $\mathrm{X}$ & $\mathrm{X}$ \\
\hline Desmodium incanum & & $X$ & $\mathrm{X}$ & & $\mathrm{X}$ \\
\hline Eriosema campestre & & & $\mathrm{X}$ & $\mathrm{X}$ & \\
\hline Eriosema crinitum & & $\mathrm{X}$ & $X$ & $\mathrm{X}$ & \\
\hline Periandra heterophylla & $\mathrm{X}$ & & $\mathrm{X}$ & & \\
\hline Zornia latifolia & $\mathrm{X}$ & $\mathrm{X}$ & $\mathrm{X}$ & $\mathrm{X}$ & \\
\hline \multicolumn{6}{|l|}{ LYTHRACEAE } \\
\hline Cuphea carthagenensis & $\mathrm{X}$ & $\mathrm{X}$ & $\mathrm{X}$ & $\mathrm{X}$ & $\mathrm{X}$ \\
\hline \multicolumn{6}{|l|}{ MALVACEAE } \\
\hline Pavonia communis & & & $\mathrm{X}$ & $\mathrm{X}$ & \\
\hline Sida cordifolia & $\mathrm{X}$ & $\mathrm{X}$ & $\mathrm{X}$ & $\mathrm{X}$ & \\
\hline Sida rhombifolia & $X$ & $X$ & $\mathrm{X}$ & $\mathrm{X}$ & $\mathrm{X}$ \\
\hline Waltheria communis & & & $\mathrm{X}$ & $\mathrm{X}$ & \\
\hline \multicolumn{6}{|l|}{ ORCHIDACEAE } \\
\hline *Oeceoclades maculata & $X$ & $X$ & $\mathrm{X}$ & $\mathrm{X}$ & \\
\hline
\end{tabular}




\begin{tabular}{|c|c|c|c|c|c|}
\hline Família/Subfamília/Espécie & Amazônia & Caatinga & Cerrado & Mata Atlântica & Pampa \\
\hline \multicolumn{6}{|l|}{ POACEAE } \\
\hline Andropogon fastigiatus & $\mathrm{X}$ & $\mathrm{X}$ & $\mathrm{X}$ & & \\
\hline Aristida riparia & $\mathrm{X}$ & $\mathrm{X}$ & $\mathrm{X}$ & $\mathrm{X}$ & \\
\hline Aristida setifolia & & $\mathrm{X}$ & $\mathrm{X}$ & & \\
\hline Axonopus pressus & $\mathrm{X}$ & $\mathrm{X}$ & $\mathrm{X}$ & $\mathrm{X}$ & \\
\hline${ }^{*}$ Digitaria insularis & $\mathrm{X}$ & $\mathrm{X}$ & $\mathrm{X}$ & $\mathrm{X}$ & \\
\hline Eragrostis polytricha & $\mathrm{X}$ & $\mathrm{X}$ & $\mathrm{X}$ & $\mathrm{X}$ & \\
\hline *Hyparrhenia rufa & $\mathrm{X}$ & $\mathrm{X}$ & $\mathrm{X}$ & $\mathrm{X}$ & \\
\hline Ichnanthus procurrens & $\mathrm{X}$ & $\mathrm{X}$ & $\mathrm{X}$ & $\mathrm{X}$ & \\
\hline *Melinis minutiflora & $\mathrm{X}$ & $\mathrm{X}$ & $\mathrm{X}$ & & \\
\hline Paspalum glaucescens & & & $\mathrm{X}$ & $\mathrm{X}$ & $\mathrm{X}$ \\
\hline *Urochloa brizantha & & $\mathrm{X}$ & $\mathrm{X}$ & $\mathrm{X}$ & $\mathrm{X}$ \\
\hline \multicolumn{6}{|l|}{ SOLANACEAE } \\
\hline Solanum sisymbrifolium & $\mathrm{X}$ & $\mathrm{X}$ & $\mathrm{X}$ & $\mathrm{X}$ & $\mathrm{X}$ \\
\hline \multicolumn{6}{|l|}{ TURNERACEAE } \\
\hline Piriqueta corumbensis & & & $\mathrm{X}$ & & \\
\hline
\end{tabular}

(1993) e Mimosa em formações abertas do Cerrado (Filgueiras 2002) e em campo sujo (Munhoz \& Felfili 2004). Comparando os dados do presente estudo com os obtidos por Cristaldo (dados não publicados) e Pott et al. (2006), observamos oito e 16 espécies em comum, respectivamente.

Segundo Coutinho (1978) o estrato herbáceosubarbustivo é constituído predominantemente por espécies perenes heliófilas, e é mal representado no cerradão, devido a intolerância ao sombreamento. A riqueza verificada para o estrato herbáceo no presente estudo, está provavelmente relacionada ao estabelecimento de espécies heliófilas nas clareiras e na borda, onde há maior incidência de luz.

Espécies hemicriptófitas, caméfitas, terófitas e geófitas decrescem nas fisionomias densas do Cerrado, sendo que a raridade das terófitas e geófitas no cerradão deva-se possivelmente à exigência de elevados níveis de luminosidade (Meira Neto et al. 2007). De fato, no presente estudo, as espécies hemicriptófitas predominaram e as terófitas e geófitas foram pouco representadas e restritas à borda ou clareiras mais amplas com alta incidência de luz.

A síndrome autocórica teve destaque entre os estratos herbáceo e subarbustivo em campo limpo, campo sujo, campo cerrado e cerrado s.s. do Parque Nacional das Emas, GO (Batalha \& Martins 2004), à semelhança do presente estudo.

Conforme avaliado, as espécies constatadas não são exclusivas do Cerrado, o que corrobora a hipótese de que o mesmo recebe influência de outros domínios brasileiros (Prado et al. 1992, Oliveira Filho \& Ratter
1995). O cerradão da RPPN urbana estudada é rico em espécies herbáceas, e apesar da antropização ainda conserva elementos da flora do Cerrado, da Mata Atlântica, da Caatinga e do Pampa.

\section{Agradecimentos}

Aos botânicos Ana Cristina Cristaldo, Fábio Matos Alves, Geciani Miriam da Silva, Leila Carvalho Costa, Vali Joana Pott, Arnildo Pott, Inês Cordeiro, José Francisco Montenegro Valls, Matías Morales e Osmar Ribas pela confirmação das identificações botânicas. À FUNDECT pelo financiamento do projeto Fauna Antófila. Ao CNPq pela concessão da bolsa de Iniciação Científica à primeira autora. À CAPES pela bolsa PRODOC concedida à segunda autora entre 2006 e 2009.

\section{Literatura citada}

APG (Angiosperm Phylogeny Group) III. 2009. An update of the Angiosperm Phylogeny Group classification for the orders and families of flowering plants: APG III. Botanical Journal of Linnean Society 161: 105-121.

Araújo, G.M., Barbosa, A.A.A., Arantes, A.A. \& Amaral, A.F. 2002. Composição florística de veredas no município de Uberlândia, MG. Revista Brasileira de Botânica 25: 475-493.

Batalha, M.A. \& Mantovani, W. 2001. Floristic composition of the Cerrado in the Pé-de-Gigante reserve (Santa Rita do Passo Quatro, Southeastern Brazil). Acta Botanica Brasilica 15:284-304.

Batalha, M.A. \& Martins, F.R. 2002. Life-form spectra of Brazilian cerrado sites. Flora 197: 452-460. 
Batalha, M.A. \& Martins, F.R. 2004. Reproductive phenology of the cerrado plant community in Emas National Park (central Brazil). Australian Journal of Botany 52:149-161

Batalha, M.A., Mantovani, W. \& Mesquita Júnior, H.N. 2001. Vegetation structure in Cerrado Physiognomies in southEastern Brazil. Brazilian Journal Biology 61: 475-483.

Carvalho, D.A. 1993. Espécies herbáceas e subarbustivas ocorrentes em cerrados do sudoeste de Minas Gerais. Ciência e Prática 17: 162-170.

Coutinho, L.M. 1978. O conceito de Cerrado. Revista Brasileira de Botânica 7: 17-23.

Embrapa-CNPGC. 1985. BoletimAgrometeorológico. Empresa Brasileira de Pesquisa Agropecuária, Campo Grande.

Filgueiras, T.S. 2002. Herbaceous plant communities. In: P.S. Oliveira \& J.R. Marquis (eds.). The cerrados of Brazil: Ecology and natural history of a neotropical savanna. Columbia University Press, New York, pp. 121-139.

Flora do Brasil. 2010. Lista de Espécies da Flora do Brasil. Jardim Botânico do Rio de Janeiro. http:// floradobrasil.jbrj.gov.br/2010/ (acesso em 10.06.2010).

Furley, P.A. \& Ratter J.A. 1988. Soil resources and plant communities of the central Brazilian cerrado and their development. Journal Bio Geografic 15: 96-108.

Guedes-Bruni, R.R., Morim, M.P., Lima, H.C. \& Silvestre, L.S. 2002. Inventário florístico. In: L. da S. Sylvestre \& M.M.T. Rosa (orgs.). Manual metodológico para estudos botânicos na Mata Atlântica. Seropédica, Rio de Janeiro, pp. 24-49.

Joly, C.A., Aidar, M.P.M., Klink, C.A., McGrath, D.G., Moreira,A.G., Moutinho, P., Nepstad, D.C., Oliveira, A., Pott, A., Rodal, M.J.N. \& Sampaio, E.V.S.B. 1999. Evolution of the Brazilian phytogeography classification systems: Implications for biodiversity conservation. Ciência e Cultura Journal of the Brazilian Association for the advancement of Science 51: 331-348.

Köppen, W. 1931. Grundriss der Klimakunde. 2 ed. W. de Gruytu, Berlin.

Mantovani, W. \& Martins, F.R. 1993. Florística do Cerrado na Reserva Biológica de Moji Guaçu, SP. Acta Botanica Brasilica 7: 33-59.

Meira Neto, J.A.A., Martins, F.R. \& Valente, G.E. 2007. Composição florística e espectro biológico na Estação Ecológica de Santa Bárbara, Estado de São Paulo, Brasil. Revista Árvore 31: 907-922.

Mendonça, R.C., Felfili,J.M., Walter, B.M.T., Silva Júnior, M.C., Rezende,A.V., Filgueiras, T.S. \& Nogueira, P.E.N. \& Fagg, C.W.2008. Flora vascular do Cerrado. Checklist com 12.356 espécies. In: S.M. Sano, S.P. Almeida \& J.F. Ribeiro (eds.). Cerrado: ambiente e flora. v.2. Embrapa Cerrados, Embrapa Informação Tecnológica, Brasília, pp. 421-443.

Mendonça, R.C., Filgueiras, T.S. \& Fagg, C.W. 2007. Análise florística da Chapada dos Veadeiros. In: J.M. Felfili, A.V. Rezende \& M.C. Silva Júnior (orgs.). Biogeografia do Bioma Cerrado: Vegetação e solos da Chapada dos Veadeiros. Editora Universidade de Brasília, Finatec, Brasília, pp. 119-124.
Müller-Dombois, D., Ellenberg, H. 1974.Aims and methods of vegetation ecology. John Wiley \& Sons, New York.

Munhoz, C.B.P. \& Felfili, J.M. 2004. Fitossociologia do componente herbáceo-subarbustivo de uma área de campo sujo no Distrito Federal, Brasil. Acta Botanica Brasilica 29: 1823-1832.

Munhoz, C.B.R. \& Felfili, J.M. 2005. Fenologia do estrato herbáceo-subarbustivo de uma comunidade de campo sujo na Fazenda Água Limpa no Distrito Federal, Brasil. Acta Botanica Brasilica 19: 979-988.

Munhoz, C.B.R. \& Felfili, J.M. 2006. Fitossociologia do estrato herbáceo-subarbustivo de uma área de campo sujo no Distrito Federal, Brasil. Acta Botanica Brasilica 20: 671-685.

Oliveira-Filho, A.T. \& Ratter, J.A. 1995. A study of the origin of central Brazilian Forests by the analysis of plant species distribution patterns. Edinburgh Journal of Botany 52: 141-194.

Pott, A., Pott, V.J., Sciamarelli, A., Sartori, A.L.B., Resende, U.M., Scremin-Dias, E., Jacques, E.L., Aragaki, S., Nakajima, J.N., Romero, R., Cristaldo, A.C.M. \& Damasceno-Junior, G.A. 2006. Inventário das Angiospermas no Complexo Aporé-Sucuriú. In: T.C.S. Pagotto \& P.R. Souza (orgs.). Biodiversidade do Complexo Aporé-Sucuriú - Subsídios à conservação e ao manejo do Cerrado. UFMS, Campo Grande, pp. 45-66.

Prado, D.E., Gibbs, P.E., Pott,A. \& Pott, V.L. 1992. The ChacoPantanal transition in southern Mato Grosso, Brazil. In: P.A Furley, J. Proctor \& J.A. Ratter (eds.). Nature and Dynamics of Forests-Savana Boundaries, Chaman \& Hall, London, pp. 461-470.

Repams. 2008. Associação de Proprietários de Reservas Particulares de Mato Grosso do Sul. http:// www.repams.org.br/rppns.php? cod=11. (Acesso em 13.12.2008).

Rodrigues, M.T. 2005. A biodiversidade dos cerrados: conhecimento atual e perspectivas, com uma hipótese sobre o papel das matas de galerias na troca faunística durante ciclos climáticos. In: A. Scariot, J.C. Sousa-Silva \& J.M. Felfili (orgs.). Cerrado: Ecologia, Biodiversidade e Conservação. Ministério do Meio Ambiente, Brasília, pp. 235-246.

Rossato, D.R., Toniato, M.T.ZU. \& Durigan, G. 2008. Flora fanerogâmica não-arbórea do cerrado na Estação Ecológica de Assis, Estado de São Paulo. Revista Brasileira de Botânica 31: 409-424.

Tannus, J.L.S. \& Assis, M.A. 2004. Composição de espécies vasculares de campo sujo e campo úmido em área de cerrado, Itirapina - SP, Brasil. Revista Brasileira de Botânica 27: 489-506.

Van der Pijl, L. 1982. Principles of dispersal in higher plants. Springer-Verlag, New York. 